\title{
UAV generalized longitudinal model for autopilot controller designs
}

\author{
Eduard Bertran \\ Castelldefels School of Telecommunications and Aerospace EngineeringTerrassa School of Industrial, Aerospace and \\ Audiovisual Engineering Department of Signal Theory and Communications, Universitat Politècnica de Catalunya, Barcelona, Spain, and \\ Paula Tercero and Alex Sànchez-Cerdà \\ Castelldefels School of Telecommunications and Aerospace Engineering Department of Signal Theory and Communications, \\ Universitat Politècnica de Catalunya, Barcelona, Spain
}

\begin{abstract}
Purpose - This paper aims to overcome the main obstacle to compare the merits of the different control strategies for fixed-wing unmanned aerial vehicles (UAVs) to assess autopilot performances. Up to now, the published studies of control strategies have been carried out over disperse models, thus being complicated, if not impossible, to compare the merits of each proposal. The authors present a worked benchmark for autopilots studies, consisting of generalized models obtained by merging UAVs' parameters gathered from selected literature (journals) with other parameters directly obtained by the authors to include some relevant UAVs whose models are not provided in the literature. To obtain them it has been used a dedicated software (from U.S. Air Force).

Design/methodology/approach - The proposed models have been constructed by averaging both the main aircraft defining parameters (model derivatives) and pole-zero locations of longitudinal transfer functions. The suitability of the used methodologies has been checked from their capability to fit the short period and the phugoid modes. Previous analytical model arrangement has been required to match a uniform set of parameters, as the inner state variables are neither the same along the different published models nor between the additional models the authors have here contributed. Besides, moving models between the space state representation and transfer function is not just a simple averaging process, as neither the parameters nor the model orders are the same in the different published works. So, the junction of the models to a common set of parameters requires some residual's computation and transient responses assessment (even Fourier analysis has been included to preserve the dominance of the phugoid) to keep the main properties of the models. The least mean squares technique has been used to have better fittings between SISO model parameters with state-space ones.

Findings - Both the SISO (Laplace) and state-space models for the longitudinal transfer function of an "averaged" fixed-wing UAV are proposed.

Research limitations/implications - More complicated situations, such as strong wind conditions, need another kind of models, usually based on finite element method simulation. These particular models apply fluid dynamics to study aerostructural aircraft aspects, such as flutter and other aerolastic aspects, the behavior under icing conditions or other distributed parameter problems. Even some models aim to control other aspects than the autopilot, such as the trajectory prediction. However, these models are not the most suitable for the basic UAV autopilot design (early design), so they are outside the objective of this paper. Obviously, the here-considered UAVs are not all the existing ones, but the number is large enough to consider the result as a reliable and realistic representation. The presented study may be seen as a stepping stone, allowing to include other UAVs in future works.

Practical implications - The proposed models can be used as benchmarks, or as a previous step to produce improved benchmarks, in order to have a common and realistic scenario the compare the benefits of the different control actions in UAV autopilots continuously presented in the published research.

Originality/value - A work with the scope of the presented one, merging model parameters from literature with other (often referred in papers and websites) whose parameters have been obtained by the authors has been never published.
\end{abstract}

Keywords Simulation, Unmanned aerial vehicles, Control systems, Aircraft modeling, Aircraft systems, Autopilots

Paper type Research paper

\section{Introduction}

The design of control strategies to be used in autopilots has different complexity, mainly depending on the size of the unmanned aerial vehicles (UAV), the implementation feasibility and the tools used to design the control law. The most elementary is the one that just facilitates a manual

The current issue and full text archive of this journal is available on Emerald Insight at: https://www.emerald.com/insight/1748-8842.htm

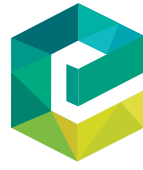

Aircraft Engineering and Aerospace Technology

(C) Emerald Publishing Limited [ISSN 1748-8842] [DOI 10.1108/AEAT-08-2020-0156] override by a remote pilot. Regarding autopilots, when the designer has not a suitable UAV model over which to develop analytical designs, the safer method is the usage of the classical proportional, integral and derivative (Poksawat et al., 2018; Chodnicki et al., 2018). A reduced set of more advanced control strategies, such as artificial neural networks (ANN) or fuzzy logic, may be also undertaken without a detailed UAV model, being often enough the classic laws of flight mechanics (i.e a kinematic analysis) to face the designs (Julian and Kochenderfe, 2017; Konar, 2019; Manerowski and Rykaczeewski, 2005; Garcia-Aunon et al., 2017; Sobolewski 
et al., 2016). The counterpart is the risk of a large training time for ANN or the incapability to consider all the possible flight disturbances or parameter variations when setting the whole set of fuzzy rules. As an ANN alternative, artificial bee colony algorithms have been used to improve some UAV performances (Konar, 2020; Konar et al., 2020).

Other advanced strategies, such as robust, adaptive, linear quadratic gaussian/linear quadratic regulator or $H_{\infty}$ have been proposed (Ghiglino and Forshaw, 2017; Bertran and Sànchez Cerdà, 2016; Sushchenko and Shyrokyi, 2015). These advanced control strategies need a better design model. Some designs allow a good computation of the controller parameters by using a dynamical model of the aircraft with reduced accuracy (i.e. transfer functions), whereas others offer better reaction to external disturbances or parameter variations at the cost of higher accuracy in the aircraft design models, usually formulated in the state-space domain.

Published research works, which show the benefits of particular and new control laws, come from simulations performed by using a specific UAV model. In addition, these models not only scatter in the kind of UAV (fixed wing, multirotor, large or short wingspan, cord, kind of rudder, etc.) but also in the following selected model parameters to design the controller: pitch, angle of attack, longitudinal and/or vertical velocity, altitude, pitch rate. Most of the published works consider both the lateral and longitudinal dynamics (Çoban, 2020; Oktay et al., 2016); being longitudinal stability models (Blakelock, 1991; Nelson, 1998) the minimum ones (alone or together with lateral) are used in new autopilot controller designs (Aliyu et al., 2015; Bertran and Sànchez Cerdà, 2016; Huang et al., 2009; Jafarov, 2006; Qazi et al., 2017; Puopolo and Jacob, 2015; Triputra et al., 2012). So, they are the first candidates to propose a step toward a generalized benchmark for results comparison.

The dependency of the results on the simulation model produces serious difficulties to assess advantages and disadvantages among the presented control laws for autopilots. As a way of dealing with this, we propose here a "benchmark model" tailored to medium size fixed-wing UAVs. To have a suitable model is the keystone to design and assess control strategies and to have a generalized one is basic when comparing their performance in the same scenario.

The model presented in this paper is obtained from actual fixed-wing UAVs with wingspans ranging from 1.2 to $6.2 \mathrm{~m}$ (the common range in professional and semiprofessional UAVs). Besides, to be representative enough, we looked for an averaged model, considering some diversities among the individual UAVs, e.g. differences in the airframe, namely, wingspan, chord, kind of rudder, tail assemblies, location of motors, kind of propulsion and so on.

To elaborate the averaged model, we have first used UAV models and parameters directly obtained from published technical papers, data sheets and such like available documentation sources. Sometimes, it has been possible to use directly the provided model parameters, whereas in other cases, the model parameters need a previous analytical arrangement to match some fixed and uniform set of parameters. And in other cases, the published models have been discarded because of the impossibility to do that. Moreover, other actual UAV longitudinal models, some of them already commercialized, have been obtained by the authors by using the U.S. Air Force (USAF) Datcom software (McDonnell Douglas Astronautics Company, 1979; Holy Cows, 2011), filling the data required in this software from UAVs structural parameters and flight conditions. This fact has allowed to consider a wider variety of UAVs structures in the final model, which will be presented in both state-space domain and as a SISO transfer function.

\section{Models of fixed-wing UAVs}

Most used aircraft longitudinal models are still based on the classical one proposed by John H. Blakelock in 1991. As it is wellknown, this model is based on the aircraft rigid body equations, using the three degree-of-freedom longitudinal equations which, along with the stability derivatives, provide the transfer functions of the aircraft for an elevator displacement input. The usual assumptions of small perturbations, mass conservation and some quasi-steady conditions are assumed. The model considers three variables: the speed in the longitudinal direction $(u)$, the angle of attack $(\alpha)$ and the pitch angle $(\theta)$, being $\alpha=\alpha_{0}+{ }^{\prime} \alpha, U=U_{0}+u$ (equilibrium and disturbed values) and ' $u=u / U$. $\mathrm{U}$ is the equilibrium velocity in the longitudinal (forward) direction (Blakelock, 1991).

After balancing steady-state aerodynamic forces and moments on the aircraft body, expanded in terms of the changes in them, the classical longitudinal equations of motion (stick fixed) are as follows:

$$
\left(\frac{m U}{S q}{ }^{\prime} \dot{u}-C_{x u} \prime u\right)+\left(-\frac{c}{2 U} C_{x \dot{\alpha}} / \dot{\alpha}-C_{x \alpha} / \alpha\right)+\left[-\frac{c}{2 U} \cdot C_{x q} \dot{\theta}-C_{w}(\cos \Theta) \theta\right]=C_{F x a}
$$

$-\left(C_{z u} \prime u\right)+\left[\left(\frac{m U}{S q}-\frac{c}{2 U} C_{z \dot{\alpha}}\right) \prime \dot{\alpha}-C_{z \alpha} \prime \alpha\right]+\left[\left(-\frac{m U}{S q}-\frac{c}{2 U} C_{z q}\right) \dot{\theta}-C_{z v}(\sin \Theta) \theta\right]=C_{F z a}$

$$
\left(-C_{m u} \prime u\right)+\left(-\frac{c}{2 U} C_{m \dot{\alpha}}{ }^{\prime} \dot{\alpha}-C_{m \alpha} \prime \alpha\right)+\left[\frac{I_{y}}{S q c} \ddot{\theta}-\frac{c}{2 U} C_{m q} \dot{\theta}\right]=C_{m a}
$$

where $m$ is the mass of the aircraft, $S$ is the wing surface, $q$ is the dynamic pressure, $c$ is the mean aerodynamic chord of the wing, $I_{Y}$ is the body inertia around $y$-axis (lateral axis). $\Theta$ is the angle between the horizontal and the $o x$-axis (forward), measured in the vertical plane and $\theta$ (being $\Theta=\Theta_{0}+\theta$ ) is the pitch angle.

These equations can be transformed to the Laplace domain to properly obtain transfer functions. To represent the excitation of the system, the elevator deflection $\delta_{e}$ and its effectiveness have been added, neglecting $C_{\mathrm{Fxa}}$. Initial conditions are equal to zero. Besides, the angles are considered small and the terms, $C_{x \dot{\alpha}}, C_{\mathrm{xq}}$ and $C_{\mathrm{mu}}$ maybe neglected. Hence, the longitudinal equations of motion become as follows:

$$
\left(\frac{m U}{S q} s-C_{x u}\right)^{\prime} u(s)-C_{x \alpha}{ }^{\prime} \alpha(s)-C_{w}(\cos \Theta) \theta(s)=0
$$


Eduard Bertran, Paula Tercero and Alex Sànchez-Cerdà

$$
\begin{gathered}
-C_{z u}{ }^{\prime} u(s)+\left[\left(\frac{m U}{S q}-\frac{c}{2 U} C_{z \dot{\alpha}}\right) s-C_{z \alpha}\right]{ }^{\prime} \alpha(s) \\
+\left[\left(-\frac{m U}{S q}-\frac{c}{2 U} C_{z q}\right) s+C_{w}(\sin \Theta)\right] \theta(s) \\
=C_{z \delta e} \delta_{e}(s) \\
\left(-\frac{c}{2 U} C_{m \dot{ }} s-C_{m \alpha}\right)^{\prime} \alpha(s)+\left(\frac{I_{y}}{S q c} s^{2}-\frac{c}{2 U} C_{m q} s\right) \theta(s) \\
=C_{m \delta e} \delta_{e}(s)
\end{gathered}
$$

\section{Models obtained from research papers}

As mentioned above, different models of UAVs may be found among the published papers, proposing control strategies for autopilots. Most part of them are based on fixed-wing UAVs, with reference wingspans ranging between 1 and $6 \mathrm{~m}$ and showing a similar geometry.

An analytical way to represent these literature models is as state-space variables. The first drawback encountered when seeking the elaboration of an averaged model is the diversity of state vectors (variables) used to build the models in different papers. A solution should be to reduce the space state models to a uniform set of variables using model reduction techniques, i.e aggregation methods (Jamshidi, 1981) reduce the system complexity preserving some characteristics of the original system. However, this kind of simplification process produces a transformed set of space-state variables, which may lose the physical meaning, a fact mandatory to better understand both the models and the performance of the designed control laws.

To keep the aircraft physical variables, as the ones used in the final model, the first idea might be to limit the individual models to those using exactly the same set of physical variables. Nevertheless, a consequence of such restriction is a drastic reduction of the number of UAVs to be included in the construction of the literature-based averaged model. To overcome this restriction, we merged models created with a similar (not equal) set of variables. Further, even the orders of the models are scarcely different. Although most of them are fourth order, there are two models that are fifth order.

These facts prevent to do a direct average of the elements of the state-space matrix, so the process to mix all the models has been to transform the state-space model into a SISO transfer function and later averaging the pole-zero locations.

In next sections, once obtained the additional Datcom models, this SISO averaged model will be enlarged with a Datcom averaged model, and, forcing the equality of some responses, the final model will be returned to the state-space domain, keeping the physical aeronautical meaning of the variables and model parameters.

The finally selected (gathered) aircrafts from literature (Triputra et al., 2012; Sufendi et al., 2013; Huang et al., 2009; Ahmed et al., 2015; Kumar and Jain, 2014; Yit and Rajendran, 2015; Aliyu et al., 2015; Persson, 2016) have wingspans ranging from 1.2 to $6.2 \mathrm{~m}$ and show slight variations in their geometry. Regarding all the models, the variables are longitudinal velocity, $u$; vertical velocity, $w$; pitch rate, $\rho$ (or $p$ ); the pitch angle, $\theta$; the angle-of-attack, $\alpha$; and the altitude of the UAV, $h$.

The common variables, which appear in all the models, are $u$, $q$ and $\theta$. So, a transfer function, relating $\theta$ (s) and $\delta \mathrm{e}(\mathrm{s})$, have been chosen, computing later their corresponding poles and zeros for all models. Notice that the poles locations are irrelevant to the kind of selected input-output variables because they are the same for all transfer functions of the same system.

In Table 1, ten published UAV models can be seen. In some cases, they are identified by the name of the UAV they are representing. When this information is unknown, the name of the model is the one of the first author of the paper in which the model has been found.

To operate with the different individual models, we have started from the following general state-space system representation (Ogata, 2010):

$$
\begin{aligned}
& \dot{x}=A x+B u \\
& y=C x+D u
\end{aligned}
$$

where $A$ and $B$ are the plant (or system) and the control matrix, respectively, $C$ is the output matrix, $D$ is the feedthrough matrix (null in the literature), $x$ is the state vector, $u$ is the input vector and $y$ is the output vector. By using the usual transformation (Ogata, 2010), we have obtained the individual longitudinal transfer functions for each model. The resulting poles and zeros are presented in Table 2 .

Short-period poles are those that produce a transient response that disappears rapidly. Whereas, phugoid poles, (dominant) are those closer to the origin, whose time constant is larger and determines the final tendency of the response.

Figure 1 shows, in the $s$-plane (Laplace), merged shortperiod and phugoid poles of all the considered models. Figure 2 shows a zoom around the origin that allows to appreciate the dominant poles (phugoid) and the lower zeros.

By averaging all the poles and zeros, in a separated framework for both the short-period poles (the ones with higher real part) and for the phugoid ones, the results are as shown in Table 3.

\section{Datcom-based models}

The models that have not been taken directly from the literature have been constructed by computing the parameters from a set of actual UAVs by using the USAF Digital Datcom software (Holy Cows, 2011). This software provides model derivatives, a fact that makes straightforward to obtain a space-state model compliant with the one introduced in equations (4)-(6). However, not all the derivatives provided by Datcom are directly comparable with the coefficients conforming the space-state models in the literature. So, the derivatives have to be transformed into a linear state space model according to the structure used in Blakelock (1991) and some additional development shown in Bertran and Sànchez Cerdà (2016).

To simplify the application of equations (4)-(6), they are converted to space-state form. To do it, the pitch rate $\dot{\theta}$ has to be included as additional variable, $\rho=\theta$. The angle $\Theta$ is supposed to be small. Hence, the used variables and parameters are the ones summarized in Table 4 . The resulting model in state space is as follows: 
Table 1 Literature models

\begin{tabular}{lcc}
\hline Model & Wingspan (m) & Variables \\
\hline BPPT WULUNG (analytical) (Triputra et al., 2012) & 6.2 & $u, w, q, \theta$ \\
BPPT WULUNG (black box) (Triputra et al., 2012) & 6.2 & $u, w, q, \theta$ \\
Sufendi et al. (2013) & - & $u, q, \theta, \alpha$ \\
Huang et al. (2009) & 2.9 & $u, q, \theta, \alpha$ \\
Ultrastick 25e (Ahmed et al., 2015) & 1.3 & $u, w, q, \theta, h$ \\
Kumar and Jain (2014) & - & $u, w, q, \theta, h$ \\
Rahimi (Yit and Rajendran, 2015) & - & $u, q, \theta, \alpha$ \\
Ultrastick 25e mini (Aliyu et al., 2015) & 1.3 & $u, w, q, \theta$ \\
Penguin B. (Persson, 2016) & 3.3 & $u, w, q, \theta$ \\
\hline
\end{tabular}

$$
\left[\begin{array}{c}
\prime \\
\dot{u} \\
\prime \dot{\alpha} \\
\dot{\theta} \\
\dot{\rho}
\end{array}\right]=A \cdot\left[\begin{array}{c}
{ }^{\prime} u \\
\prime \alpha \\
\theta \\
\rho
\end{array}\right]+B \cdot \delta_{e}
$$

where $B$ is a $4 \times 1$ matrix, and $A$ is a $4 \times 4$ matrix.

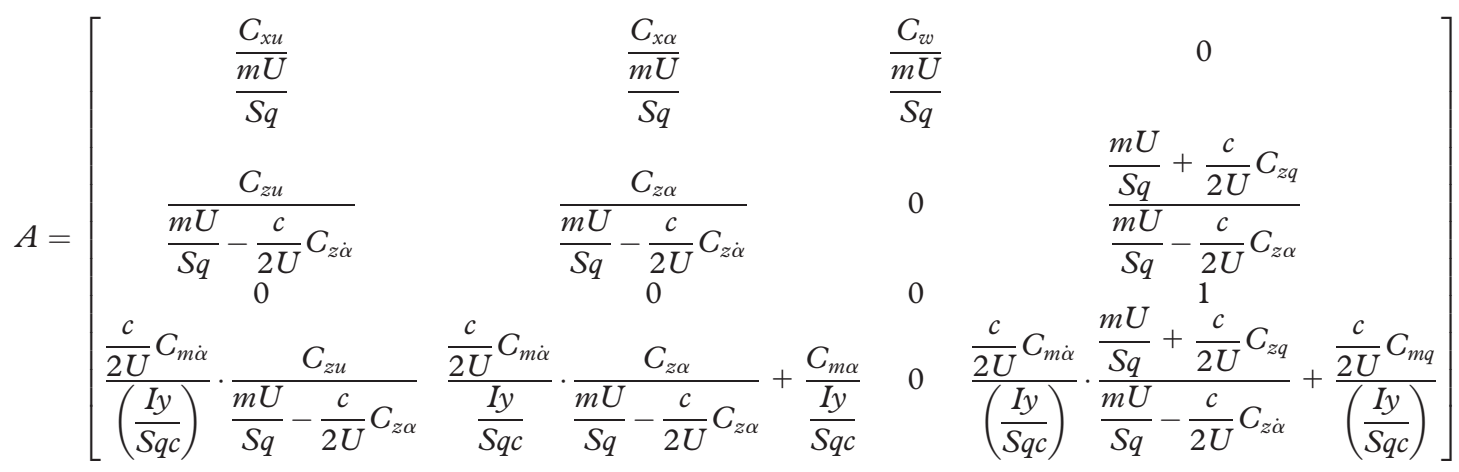

Being the model parameters defined as in previous equations (1)-(3).

The parameter $I_{\mathrm{y}}$ has been computed from the actual UAVs by adding both the aircraft body and wings inertia. The derivatives $C_{\mathrm{x}}, C_{\mathrm{w}}, C_{\mathrm{z}}$ and $C_{\mathrm{m}}$ may be computed by using Datcom.

In total, 10 UAVs have been modeled (Figure 3). These are: ALO and SIVA (INTA, 2020), Megastar (PROTECH data, 2020; Tristancho et al., 2010), Shadow (Tecktron Systems, 2009), Ant Plane (Funaki and Hirasawa, 2008), Perching (Puopolo and Jacob, 2015), Raven (AeroVironment, 2020), Taurus (Qazi et al., 2017), Brayraktar (Army Technology, 2020) and IAI (2013). ALO (span: $3.84 \mathrm{~m}$ and maximum takeoff weight, maximum take-off weight (MTOW): $55 \mathrm{~kg}$ ) and SIVA (span: $5.81 \mathrm{~m}$, maximum weight: $300 \mathrm{~kg}$ ) are UAVs developed by the Spanish National Institute for Aerospace Technology. Megastar 1.50 ARF, manufactured by Proteus, has a wingspan of $2.4 \mathrm{~m}$ and a weight of $5.9 \mathrm{~kg}$, whereas Shadow MK-1 is an experimental prototype, with $5.2 \mathrm{~m}$ of wingspan, and allowing a MTOW of $90 \mathrm{~kg}$ (dry weight of $55 \mathrm{~kg}$ ). Ant Plane is a scale model of the Grob G109 (Aeronaut company, Germany), with a length of $1.32 \mathrm{~m}$, wingspan of $2.76 \mathrm{~m}$ and height of $0.37 \mathrm{~m}$, cruising speed of $70 \mathrm{~km} / \mathrm{h}$ and $5 \mathrm{~kg}$ in take-off weight. Perching UAV was created to simulate the behavior of a bird (span: $1.4 \mathrm{~m}$, weight: $0.9 \mathrm{~kg}$ ). Raven (span: $1.4 \mathrm{~m}$, weight: $1.9 \mathrm{~kg}$ ) is a lightweight unmanned aerial system (UAS) which is the most prolific small UAS deployed with the USAF. Taurus is an UAV with a span of $1.3 \mathrm{~m}$. Bayraktar (span:2.8 m, weight: $3.5 \mathrm{~kg}$ ) is a currently operational mini UAV designed and produced by the Turkish Armed Forces for short-range reconnaissance and surveillance missions. IAI (span: $3.9 \mathrm{~m}$, weight: $104.0 \mathrm{~kg}$ ) is developed by the Israel Aerospace Industries.

Besides, two large fixed-wing UAVs were considered for incorporation into the averaged model. These were the Global Hawk (2019), with a wingspan of $35.3 \mathrm{~m}$ and the scale-sized technological demonstrator ( $24 \mathrm{~m}$ wingspan) of the Heliplat 
Table 2 Poles and zeros of the literature models

\begin{tabular}{|c|c|c|}
\hline Model & Zeros & Poles \\
\hline BPPT WULUNG (analytical) (Triputra et al., 2012) & $\begin{array}{l}-9.595 \\
-0.076\end{array}$ & $\begin{array}{c}-71.255 \\
-0.722 \\
-0.044 \pm 0.313 i\end{array}$ \\
\hline BPPT WULUNG (black box) (Triputra et al., 2012) & $\begin{array}{l}-76.331 \\
-0.419 \\
-0.056\end{array}$ & $\begin{array}{c}-2.778 \\
-0.881 \\
-0.044 \pm 0.159 i\end{array}$ \\
\hline Sufendi et al. (2013) & $\begin{array}{l}-5975 \\
-0.165\end{array}$ & $\begin{array}{l}-6.112 \pm 4.925 i \\
-0.061 \pm 0.405 i\end{array}$ \\
\hline Huang et al. (2009) & $\begin{array}{l}-3.210 \\
-0.094 \\
-0.000\end{array}$ & $\begin{array}{c}-4.936 \pm 10.259 i \\
-0.015 \pm 0.432 i \\
0.000\end{array}$ \\
\hline Ultrastick 25e (Ahmed et al., 2015) & $\begin{array}{l}-7.322 \\
-0.679 \\
-0.000\end{array}$ & $\begin{array}{c}-11.683 \pm 10.016 \\
-0.299 \pm 0.675 i \\
0.000\end{array}$ \\
\hline Kumar and Jain (2014) & $\begin{array}{c}1.886 \\
-0.152 \\
0.000\end{array}$ & $\begin{array}{c}1.994 \pm 3.785 i \\
-0.162 \pm 0.299 i \\
0.000\end{array}$ \\
\hline Rahimi (Yit and Rajendran, 2015) & $\begin{array}{l}-2.079 \\
-0.074\end{array}$ & $\begin{array}{l}-1.993 \pm 4.744 \mathrm{i} \\
-0.020 \pm 0.257 \mathrm{i}\end{array}$ \\
\hline Ultrastick 25e mini (Aliyu et al., 2015) & $\begin{array}{l}-9.046 \\
-0.785\end{array}$ & $\begin{aligned}-15.318 & \pm 13.400 \\
-0.367 & \pm 0.500 \mathrm{i}\end{aligned}$ \\
\hline Penguin B. (Persson, 2016) & $\begin{array}{l}-3.206 \\
-0.178\end{array}$ & $\begin{array}{l}-4.596 \pm 7.796 \mathrm{i} \\
-0.054 \pm 0.540 \mathrm{i}\end{array}$ \\
\hline
\end{tabular}

Figure 1 Map of pole-zero plots for the literature models

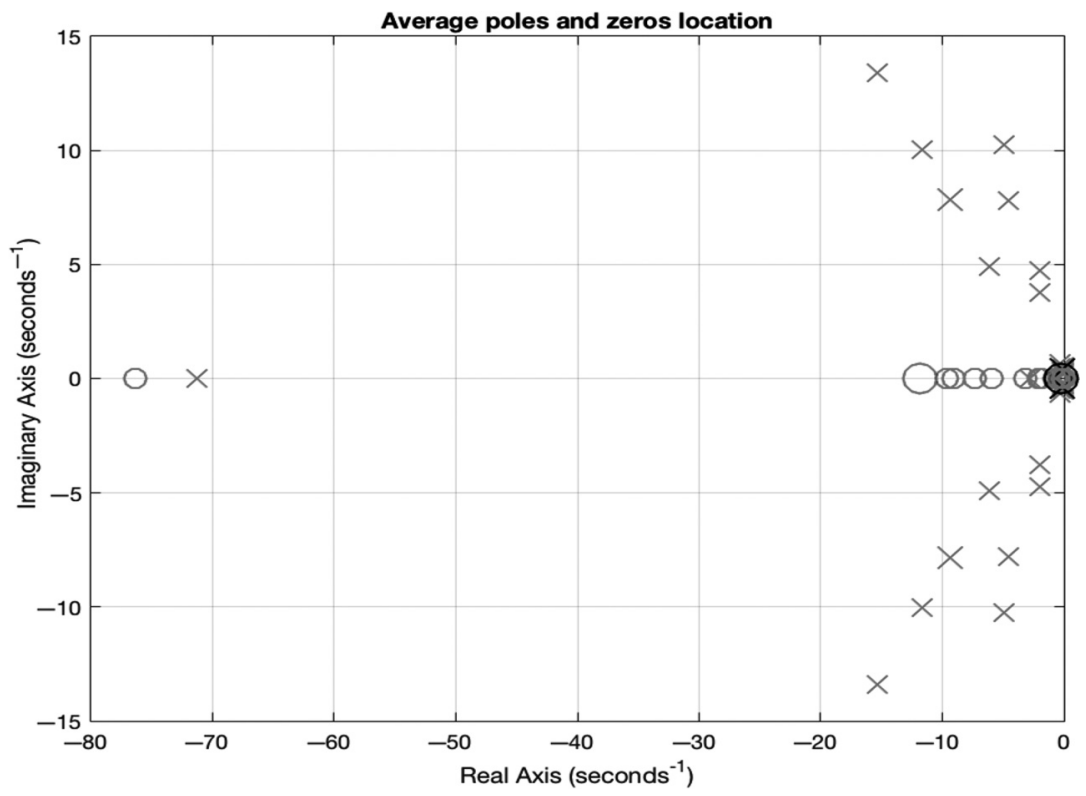

prototype (HeliNet Project,2000/2003). However, the differences among the parameters of both models compared to those of the lower wingspan models were too unbalancing. For the shake of generality of the averaged model; we decided to exclude them.

Fortunately, Datcom offers an additional output file which plots, approximately, the geometry it is using to do its calculations. So, in each case, it is possible to check that we are really modeling the desired UAV (Figure 3). When every needed data was obtained, the input files have been created, and all the derivatives required by the Blakelock model have been calculated.

\section{Derivatives average (Option 1)}

The first way of averaging the Datcom models is to compute the mean of the individual derivatives of the 10 models before 
Figure 2 Zoom at phugoid poles and lower zeros

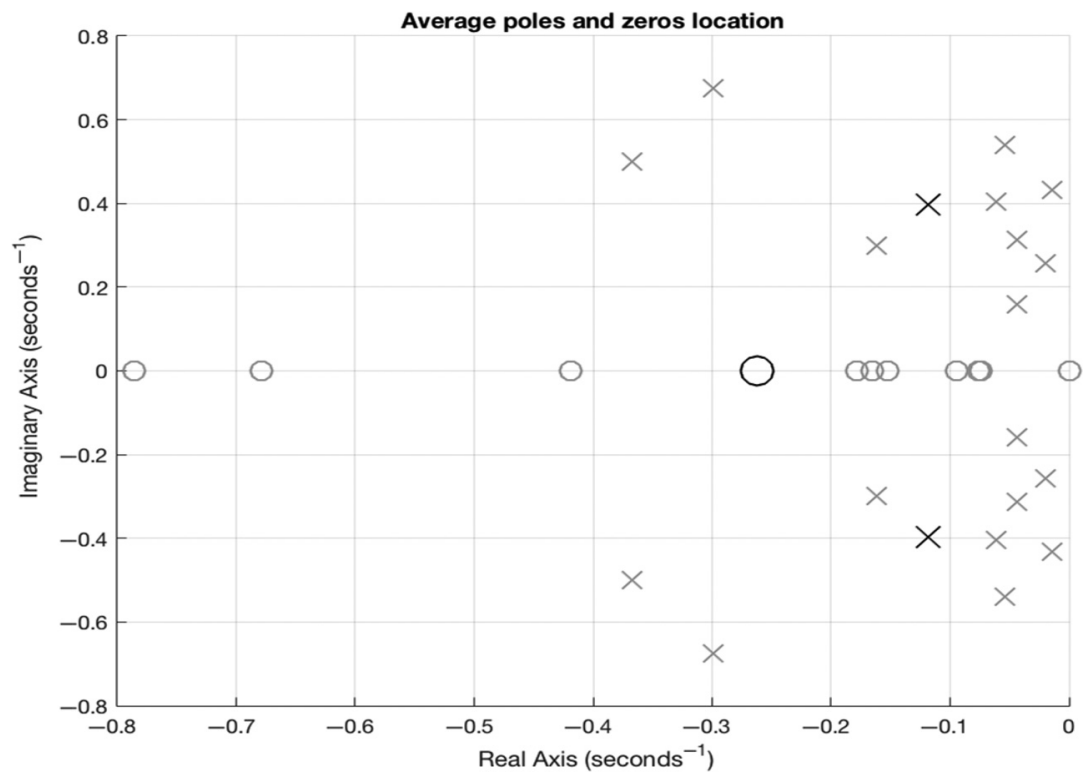

Table 3 Averaged poles and zeros from literature models

\begin{tabular}{lcc}
\hline Zeros & Short period & Phugoid \\
\hline$-11.863,-0.262$ & $-9.378 \pm 7.847 \mathrm{i}$ & $-0.118 \pm 0.398 \mathrm{i}$ \\
\hline
\end{tabular}

creating the state-space model as in equations (8)-(10). Finally, an averaged state-space system has been computed. Table 5 shows the different derivatives and their averaged value.

With these derivatives, a linear state space model is computed. The associated transfer function between $\theta$ and $\delta_{\mathrm{e}}$ has been obtained, being the poles and zeros as in Table 6 .

Regarding the poles, it is remarkable that the imaginary parts are very similar to the ones obtained with the literature models. However, the differences become bigger in the real parts, showing the Datcom model slower phugoid (poles closer to the origin) and short-period transients. Lastly, both zeros are also smaller in the Datcom model. Even though both obtained models keep qualitatively the same features: one zero closer to the origin, one bigger zero and two pairs of complex conjugated poles that can be separated as phugoid and short-period components.

\section{Poles and zeros average (Option 2)}

The second option consists in computing one model for each UAV and then to average the poles and zeros (not the derivatives) obtained for each model. The pole and zero values spread from values lower than 1 to higher than 23. In particular, the real part of the poles associated to the phugoid range from $s=-0.001$ to $s=-0.24$. This can be seen in Figures 4 and 5. Once averaged, the resulting poles and zeros are shown in Table 7.

It can be pointed out that these results have the same order of magnitude as the ones obtained in the literature models (research papers) and the values are quite similar. On the contrary, some remarkable differences appear between the location of the poles and zeros obtained with the different

Table 4 Tabulated aerodynamic variables and parameters

\begin{tabular}{|c|c|c|c|}
\hline$u$ & Disturbed longitudinal (forward) velocity & $q$ & Dynamic pressure \\
\hline $\boldsymbol{U}$ & Nominal forward velocity (equilibrium) & $c$ & Mean aerodynamic chord (MAC) of the wing, \\
\hline$w$ & Disturbed vertical velocity & $I_{Y}$ & Body inertia around $y$-axis (lateral axis) \\
\hline$\alpha$ & Disturbed angle of attack & $\delta \mathrm{e}$ & Elevator displacement \\
\hline \multirow[t]{8}{*}{$\theta$} & Pitch angle & $C_{\mathrm{x}}$ & Control and stability derivatives: \\
\hline & & $C_{\mathrm{w}}$ & $C x_{u}$ (variation of drag and thrust with $u$ ), \\
\hline & & $C_{\mathrm{z}}$ & $\boldsymbol{C}_{\boldsymbol{\alpha}}$ (lift and drag variations along $x$-axis of thrust with $u$ ), \\
\hline & & $C_{\mathrm{m}}$ & $C_{w}$ (gravity), \\
\hline & & & $C z_{u}$ (variation of normal force with $\mathrm{u}$ ), \\
\hline & & & $C z_{\alpha}$ (slope of the normal force curve), \\
\hline & & & $C m_{\alpha}$ (static longitudinal stability) and \\
\hline & & & $C m_{q}$ (damping in pitch) \\
\hline $\boldsymbol{\rho}=\dot{\boldsymbol{\theta}}$ & Pitch rate & $m$ & Mass of the aircraft \\
\hline$S$ & Wing surface & $h$ & Altitude \\
\hline
\end{tabular}


Figure 3 Datcom models

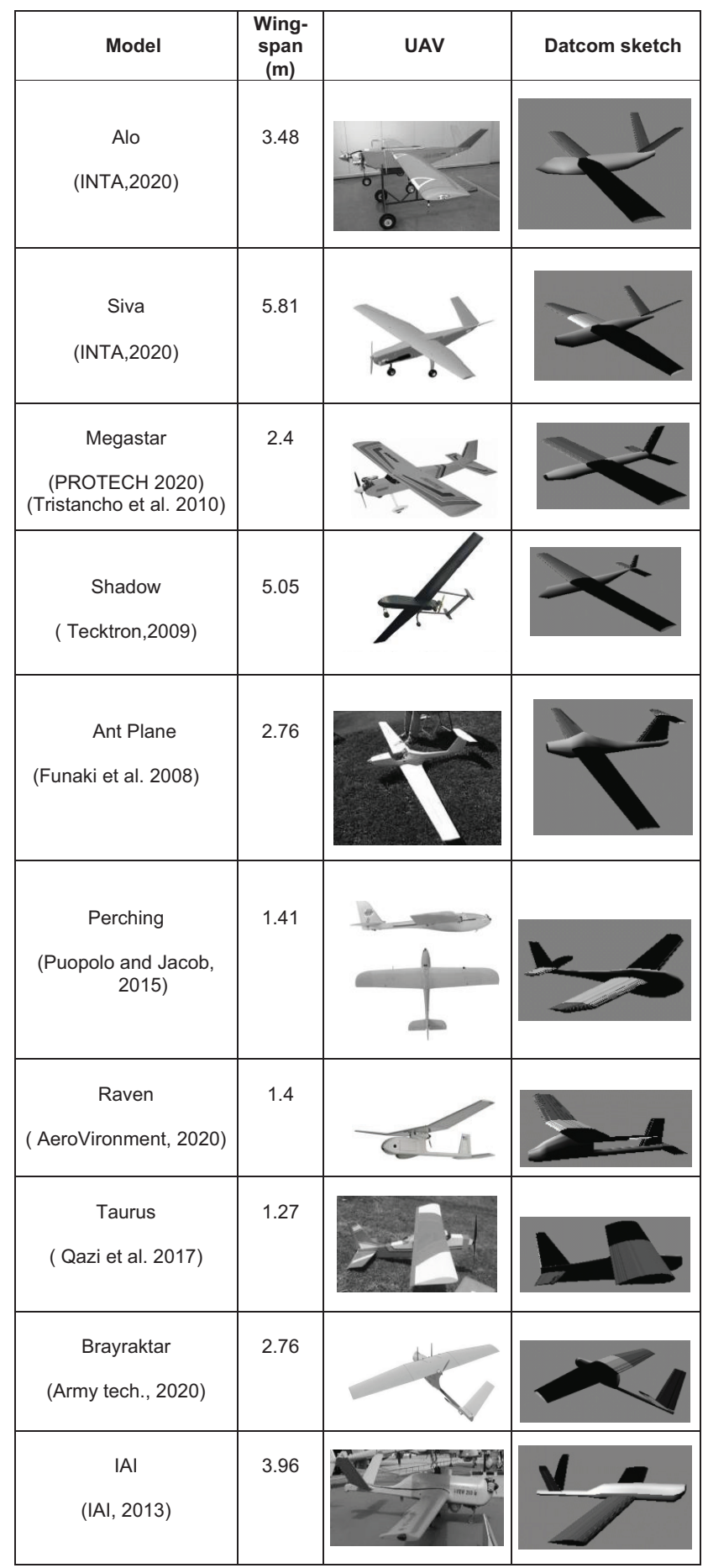

Table 5 Averaged derivatives

\begin{tabular}{lcccccccc}
\hline Derivative & $C x u$ & $C x a$ & $C w$ & $C z u$ & $C z a$ & $C z q$ & $C m a$ \\
\hline Average & -0.148 & 0.229 & -0.759 & -1.143 & -7.063 & -6.353 & -3.683 \\
Derivative & $C z a$ & $C m a$ & $C m q$ & $C m \delta e$ & $C z \delta e$ & ly \\
Average & -0.147 & -0.492 & -19.346 & -2.626 & -0.729 & 47.901 \\
\hline
\end{tabular}

Table 6 Averaged poles and zeros of the Datcom models group (Option 1)

\begin{tabular}{llc}
\hline Zeros & Short - Period & Phugoid \\
\hline$-2.253,-0.072$ & $-2.378 \pm 7.121 \mathrm{i}$ & $-0.024 \pm 0.335 \mathrm{i}$ \\
\hline
\end{tabular}

Datcom Options 1 and 2. For this reason, they are compared and assessed in the next subsection to select the most reliable model parameters.

\section{Datcom models comparison}

Concerning the two options considered above for the Datcom models, both provide final models with a similar qualitative distribution of poles and zeros, which would indicate a related behavior in both cases. However, the values of these poles and zeros are different because both methods do not achieve exactly the same result.

Nevertheless, it is easy to understand that both methods are not operating mathematically in the same way; regarding the poles, they are calculated as the eigenvalues of the $A$ matrix. Consequently, the poles (eigenvalues of Matrix $A$, represented by $\lambda$ ) have to fulfill the following equation:

$$
\operatorname{det}(\mathrm{A}-\lambda \mathrm{I})=0
$$

which implies the use of determinants. In particular, Option 1 is comparable to do the average of a whole $A$ matrix (once averaged) and later, to compute the determinant of this averaged matrix. Otherwise, Option 2 can be defined as computing the determinant of each individual $A$ matrix (one matrix for each UAV model) and then do the average of the results of the determinants. It can be understood the difference of results between Options 1 and 2 because the noncommutative operations with determinants.

Consequently, to assess both models, we have simulated the step responses of all the considered UAVs. The amplitudes have been scaled to avoid providing privileges to any individual response. In Figure 6 the averaged time response is shown.

Next step is to compute the Fast Fourier transform (FFT) of the averaged response. In Figure 7, angular frequencies are shown in the $x$-axis, and the peak of these frequencies is located at $\omega=0.6 \mathrm{rad} \mathrm{s}-1$.

To compare the frequencies found with the FFT with the actual frequencies of the averaged models, the poles that should be mainly considered are the phugoid ones because they are the more critical in aircraft stability. The damping frequencies (the imaginary parts of the roots) of the averaged systems from Options 1 and 2 (Tables 6 and 7) are as follows:

$$
\begin{aligned}
& \text { Option } 1= \pm \omega_{d}= \pm 0.335 \\
& \text { Option 2 }= \pm \omega_{d}= \pm 0.561
\end{aligned}
$$

Regarding these results, the frequency of the Datcom averaged system is better adjusted with Option 2 .

\section{Total average}

Once decided the Option 2 as the best average of the subset of models obtained by using the Datcom software, the next step is to do the final mean between the Datcom and the literature models. The final poles and zeros are summarized in Table 8 . 
Figure 4 Short-period poles and greater zeros

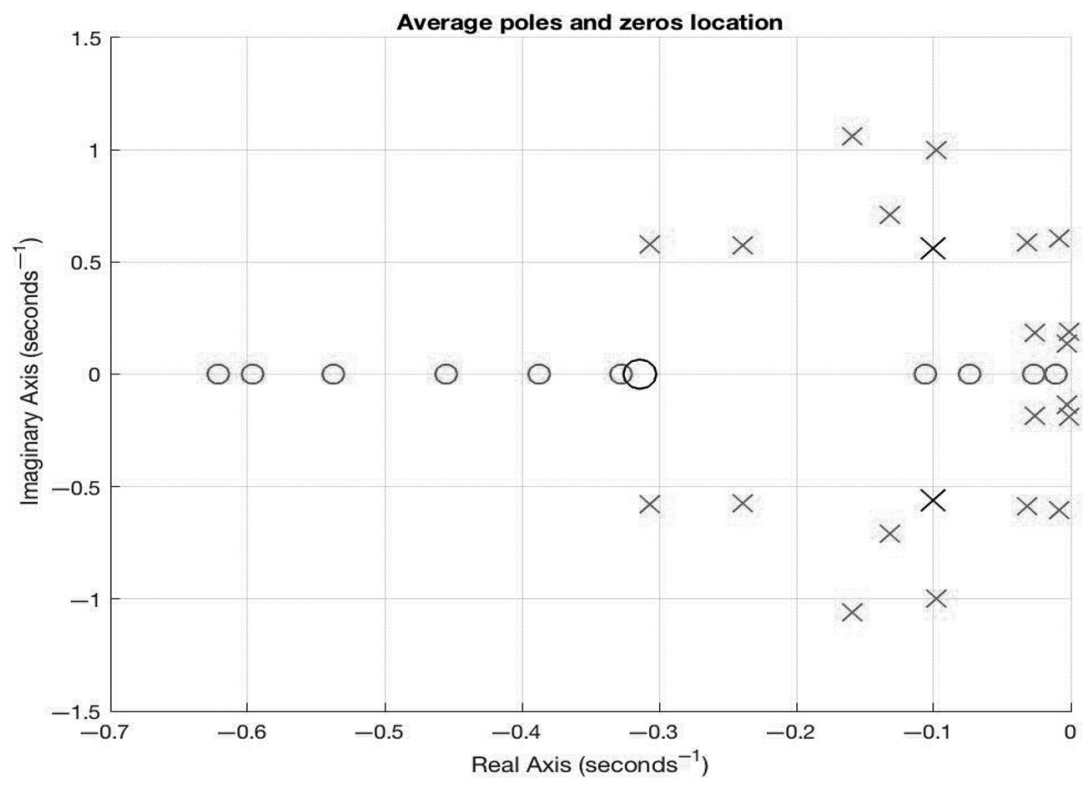

Figure 5 Zoom at phugoid poles and lower zeros

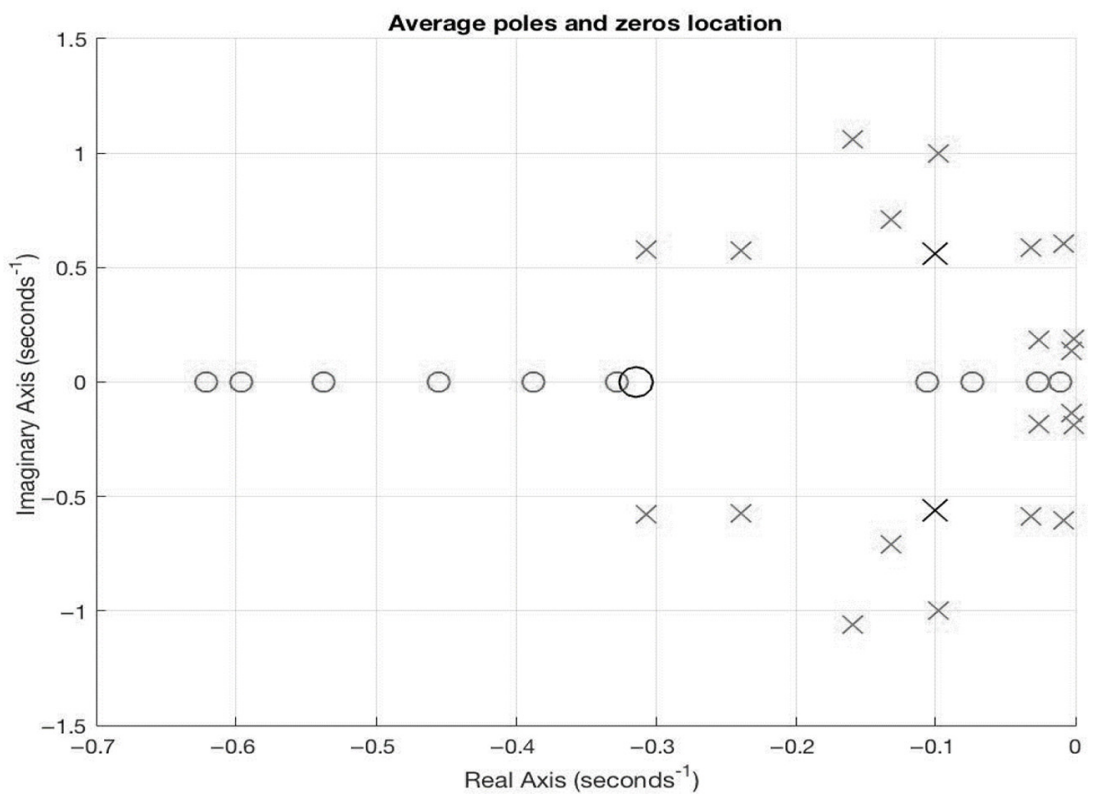

Table 7 Averaged poles and zeros of the Datcom models (Option 2)

\begin{tabular}{lcc}
\hline Zeros & Short period & Phugoid \\
\hline$-9.256,-0.314$ & $-9.789 \pm 12.065 \mathrm{i}$ & $-0.101 \pm 0.561 \mathrm{i}$ \\
\hline
\end{tabular}

The transfer function of the model is as follows:

$$
\frac{\theta(s)}{\delta_{e}(s)}=K \cdot \frac{s^{2}+11.08 s+3.438}{s^{4}+18.53 s^{3}+184.9 s^{2}+41.7 s+41.27}
$$

The value of $K$ will be computed below, by averaging the steady-state responses of the literature models, with the Datcom models.

Once obtained the SISO transfer function in equation (12), the next step is to obtain the equivalent state-space model. By using the same variables and models of equations (9) and (10), the averaged $B$ and $A$ matrices from the individual Datcom models are as follows: 
Figure 6 Averaged step response

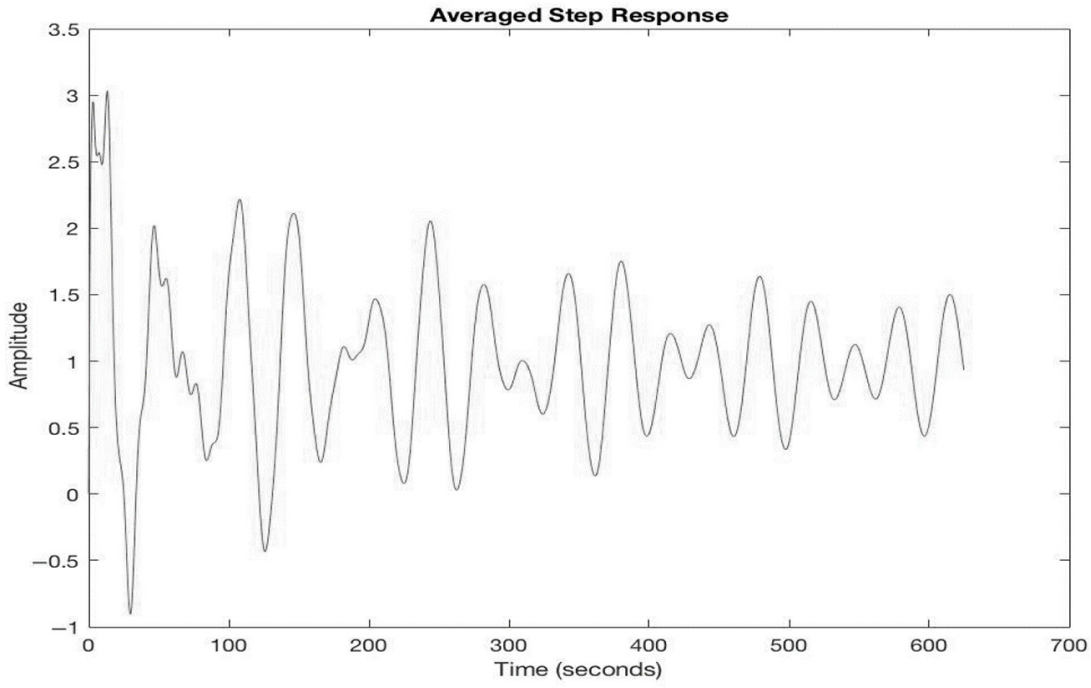

Figure 7 Fast Fourier transform of the averaged step response

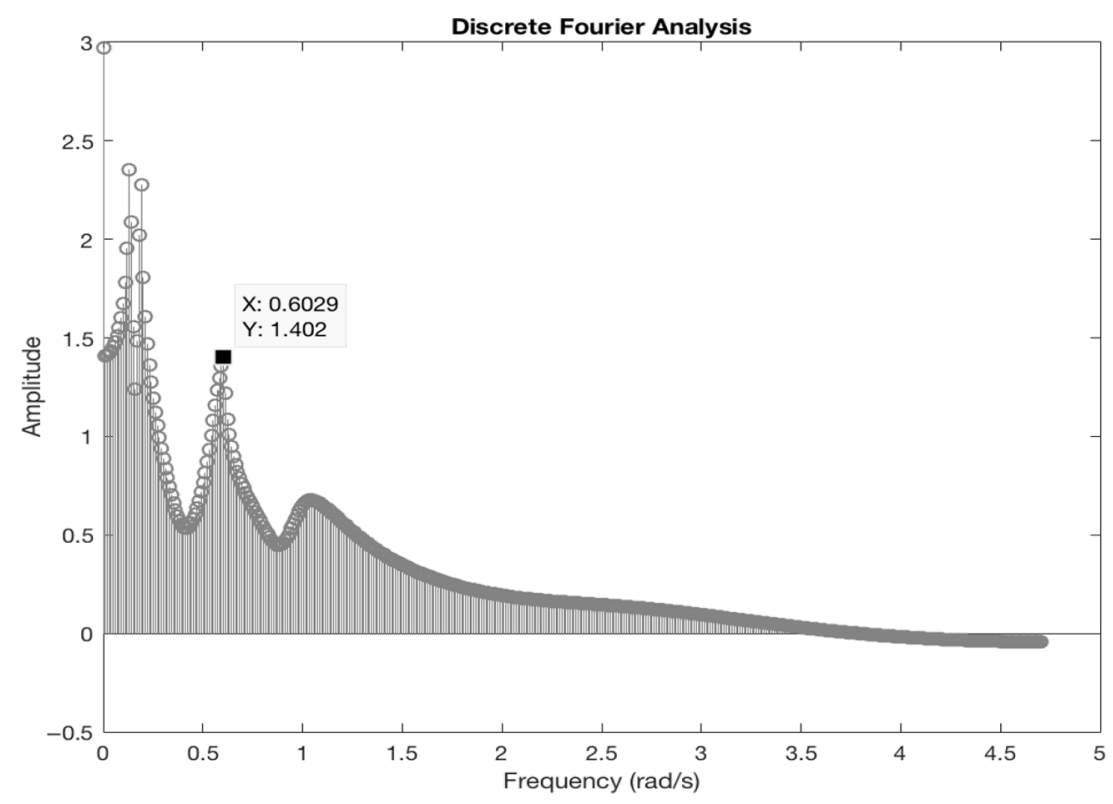

Table 8 Averaged poles and zeros of the final model

\begin{tabular}{lcc}
\hline Zeros & Short period & Phugoid \\
\hline$-10.761,-0.320$ & $-9.162 \pm 9.846 \mathrm{i}$ & $-0.104 \pm 0.466 \mathrm{i}$ \\
\hline & $B=\left(\begin{array}{c}0 \\
-0.3008 \\
0 \\
-98.6580\end{array}\right)$
\end{tabular}

$$
A=\left(\begin{array}{cccc}
-0.2397 & 0.3447 & -0.4108 & 0 \\
-1.9045 & -10.6946 & 0 & 0.9413 \\
0 & 0 & 0 & 1 \\
0.4573 & -250.5130 & 0 & -8.8440
\end{array}\right)
$$

Starting from these equations, the objective is to adjust the coefficients of Matrix $A$ to fit its eigenvalues to the poles shown in the SISO model of equation (12). This adjustment will be made by using the LMS (least mean squares, Dixitt and Nagaria, 2017). 


\section{Least mean squares adjustment of the steady-state model parameters}

To adjust the parameters in equation (10), the matrix (14) has been weighted, around the unity, by the above-mentioned derivatives. Hence, to start the optimization process, matrix $A$ has been as follows:

$A=\left(\begin{array}{cccc}-0.2397 \cdot \mathrm{Cxu} & 0.3447 & -0.4108 \cdot \mathrm{Cw} & 0 \\ -1.9045 \cdot \mathrm{Czu} & -10.6946 \cdot \mathrm{Cz} \alpha & 0 & 0.9413 \\ 0 & 0 & 0 & 1 \\ 0.4573 \cdot \mathrm{Czu} & -250.5130 \cdot \mathrm{Cz} \alpha & 0 & -8.8440\end{array}\right)$

The LMS algorithm is used to minimize the error between the step response of the SISO model and the one of the steady-state model. The values of the derivatives have been modified as follows according to the LMS rule:

$$
w[n+1]=w[n]+\mu e[n] u[n]
$$

where $n$ is the current computer iteration, $e$ is the error between both step responses, $u$ is the SISO model step response and $\mathrm{w}$ is the weights to iteratively adjust the derivatives (i.e when adjusting $\boldsymbol{C w}$ the element in equation (13) of the previous $A$ matrix becomes $-\mathbf{0 . 4 1 0 8} \cdot \mathbf{C w} \cdot \mathbf{w})$. The value of the coefficient $\mu$ has been set to $10^{-5}$. The algorithm converged to the following:

$$
A=\left(\begin{array}{cccc}
-0.2145 & 0.3447 & -0.3759 & 0 \\
-1.0475 & -9.5396 & 0 & 0.9413 \\
0 & 0 & 0 & 1 \\
0.2515 & -102.7905 & 0 & -8.8440
\end{array}\right)
$$

with eigenvalues (poles) and zeros as shown in Table 9.

Table 9 Poles and zeros of the steady-state model

\begin{tabular}{lcc}
\hline Zeros & Short period & Phugoid \\
\hline$-9.1859-0.2548$ & $-9.1947 \pm 9.8397 \mathrm{i}$ & $-0.1044 \pm 0.4661 \mathrm{i}$ \\
\hline
\end{tabular}

The response for a unity step set-point in the elevators is shown in Figure 8.

By merging the literature models with the ones obtained with Datcom, the value of the gain $K$ in equation (12) is as follows:

$K=-5.6$

The negative sign is to be coherent with the National Advisory Committee for Aeronautics convention; a positive elevator (down elevator, stick forward) deflection produces a negative pitch.

\section{Conclusion}

A longitudinal model for small fixed-wing UAVs has been obtained, merging both already published data (once processed) and data computed and assessed by the authors from different UAV prototypes, most of them already commercialized. The objective is to propose a benchmark model to check (i.e. to simulate) control strategies for autopilots, thus making easier the results comparison among different control strategies. The final model combines results considering both the UAV model parameters (derivatives) and the dynamics from the pole-zero locations. Both the SISO and state-space model have been presented. All the models have been obtained from the classical assumptions of constant weight and atmosphere, the most usual scenario.

More complicated situations, such as strong wind conditions, need another kind of models, usually based on finite element method simulation. These particular models apply fluid dynamics to study aerostructural aircraft aspects, such as flutter and other aerolastic aspects, the behavior under icing conditions or other distributed parameter problems. Even some models aim to control other aspects than the autopilot, such as the trajectory prediction. A work in that way, using a probabilistic multiple model may be seen in Jilkov et al. (2019). However, these models are not the most suitable for facing the basic UAV autopilot design (early design), which is the objective of this paper.

Obviously, the here-considered UAVs are not all the existing ones, but the number is large enough to consider the result as a reliable and realistic representation. The presented study may be seen as a stepping stone, allowing to include other UAVs in future works.

Figure 8 Step response of the averaged model

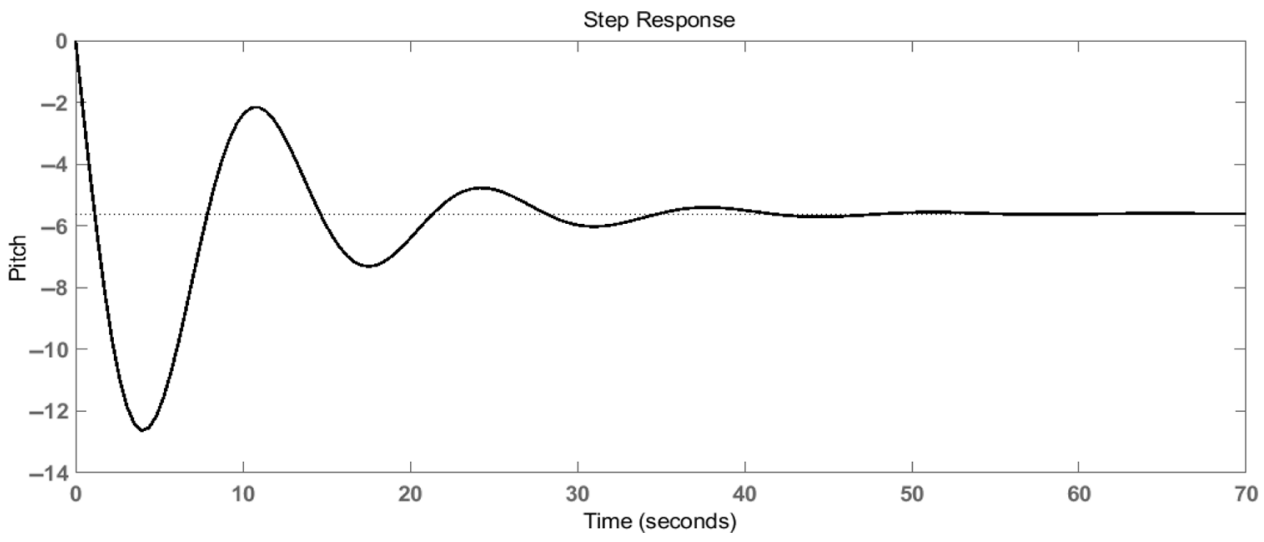




\section{References}

AeroVironment (2020), "UAS: rq-11b raven", available at: www.avinc.com/uas/view/raven

Ahmed, E.A., Hafez, A., Ouda, A.N., Ahmed, H.E.H. and Abd-Elkader, H.M. (2015), "Modelling of a small unmanned aerial vehicle", International Advances in Robotics and Automation, Vol. 4 No. 1.

Aliyu, B.K., Osheku, C.A., Okeke, P.N., Opara, F.E. and Okere, B.I. (2015), "Oscillation analysis for longitudinal dynamics of a fixed-wing UAV using PID control design", Advances in Research, Vol. 5 No. 3, pp. 1-13.

Army Technology (2020), "Bayraktar mini unmanned aerial vehicle", available at: www.army-technology.com/projects/ bayraktar-uav/

Bertran, E. and Sànchez Cerdà, A. (2016), "On the tradeoff between electrical power consumption and flight performance in fixed-wing UAV autopilots", IEEE Transactions on Vehicular Technology, Vol. 65 No. 11, pp. 8832-8840.

Blakelock, J.H. (1991), Automatic Control of Aircraft and Missiles, 2nd ed., John Wiley \& Sons, Inc.

Chodnicki, M., Bartnik, K., Nowakowski, M. and Kowaleczko, G. (2018), "Design and analysis of a feedback loop to regulate the basic parameters of the unmanned aircraft", Aircraft Engineering and Aerospace Technology, Vol. 92 No. 3, pp. 318-328.

Çoban, S. (2020), "Autonomous performance maximization of research-based hybrid unmanned aerial vehicle", Aircraft Engineering and Aerospace Technology, Vol. 92 No. 4, pp. 645-651.

Dixitt, S. and Nagaria, D. (2017), "LMS adaptive filters for noise cancellation: a review", International fournal of Electrical and Computer Engineering (IFECE), Vol. 7 No. 5, pp. 2520-2529.

Funaki, M. and Hirasawa, N. and the Ant-Plane Group (2008), "Outline of a small un-manned aerial vehicle (AntPlane) designed for Antarctic research", National Institute of Polar Research.

Garcia-Aunon, P., Santos, M. and De la Cruz, J.M. (2017), "Parameter selection based on fuzzy logic to improve UAV path-following algorithms", fournal of Applied Logic, Vol. 24, pp. 62-75, part B.

Ghiglino, P. and Forshaw, J.L. (2017), "HEX2OQTAL: translational optimal control exploiting quaternion error dynamics", IEEE Transactions on Aerospace and Electronic Systems, Vol. 53 No. 3, pp. 1181-1195.

Global Hawk (2019), “Global security org”, available at: www. globalsecurity.org/intell/systems/global_hawk-pics.htm

HeliNet Project (2000/2003), "European framework program 5".

Holy Cows (2011), Datcom + Pro User's Manual-Version 3.0, Holy Cows, Orlando.

Huang, C., Shao, Q., Jin, P., Zhu, Z. and Zhang, B. (2009), "Pitch attitude controller design and simulation for a small unmanned aerial vehicle", International Conference on Intelligent Human-Machine Systems and Cybernetics, Hangzhou, pp. 58-61.

IAI (2013), "Israel aerospace industries", available at: www.iai.co.il INTA (2020), "National institute for aerospace technology (Spain). Technical information: high technology programs", Unmanned Aircraft.
Jafarov, E.M. (2006), "H inf. Loop shaping robust control vs. classical PI (D) control: a case study on the longitudinal dynamics of Hezarfen UAV", Proc. 2nd WSEAS Int. Conf. Dyn. Syst. Control, pp. 105-110.

Jamshidi, M. (1981), "An overview on the aggregation of largescale systems", IFAC Proceedings Volumes, Vol. 14 No. 2, pp. 1309-1314.

Jilkov, V.P., Ledet, J.H. and Li, X.R. (2019), "Multiple model method for aircraft conflict detection and resolution in intent and weather uncertainty", IEEE Transactions on Aerospace and Electronic Systems, Vol. 55 No. 2, pp. 1004-1020.

Julian, K. and Kochenderfe, M.J. (2017), "Neural network guidance for UAVs", AIAA Guidance, Navigation, and Control Conference.

Yit, K.K. and Rajendran, P. (2015), "Enhanced longitudinal motion control of UAV simulation by using P-LQR method", International fournal of Micro Air Vehicles, Vol. 7 No. 2, pp. 203-210.

Konar, M. (2019), “Redesign of morphing UAV's winglet using DS algorithm based ANFIS model", Aircraft Engineering and Aerospace Technology, Vol. 91 No. 9, pp. 1214-1222.

Konar, M. (2020), "Simultaneous determination of maximum acceleration and endurance of morphing UAV with ABC algorithm-based model", Aircraft Engineering and Aerospace Technology, Vol. 92 No. 4, pp. 579-586.

Konar, M., Turkmen, A. and Oktay, T. (2020), "Improvement of the thrust-torque ratio of an unmanned helicopter by using the ABC algorithm", Aircraft Engineering and Aerospace Technology, Vol. 92 No. 8, pp. 1133-1139.

Kumar, N. and Jain, S. (2014), "Identification, modeling and control of unmanned aerial vehicles", International Fournal of Advanced Science and Technology, Vol. 67, pp. 1-10.

McDonnell Douglas Astronautics Company (1979), The USAF Stability and Control Datcom -Volume I, User's Manual, St. Louis Division, MO.

Manerowski, J. and Rykaczeewski, D. (2005), "Modelling of UAV flight dynamics using perceptron artificial neural networks", Fournal of Theoretical and Applied Mechanics, Vol. 42 No. 2, pp. 297-307.

Nelson, R.C. (1998), Flight Stability and Automatic Control, 2nd ed., McGraw-Hill.

Ogata, K. (2010), Modern Control Engineering, 5th ed., Prentice Hall.

Oktay, T., Konar, M., Onay, M., Aydin, M. and Mohamed, M.A. (2016), "Simultaneous small UAV and autopilot system design", Aircraft Engineering and Aerospace Technology, Vol. 88 No. 6, pp. 818-834.

Persson, L. (2016), "Cooperative control for landing a fixedwing unmanned aerial vehicle on a ground vehicle", KTH, School of Electrical Engineering (thesis), Stockholm, available at: https://kth.diva-portal.org/smash/get/diva2: 930987/FULLTEXT01.pdf

Poksawat, P., Wang, L. and Mohamed, A. (2018), "Gain scheduled attitude control of fixed-wing UAV with automatic controller tuning", IEEE Transactions on Control Systems Technology, Vol. 26 No. 4, pp. 1192-1203. 
PROTECH data (2020), available at: www.galaxia.co.il/ protech.htm

Puopolo, M. and Jacob, J.D. (2015), "Model for longitudinal perch maneuvers of a fixed-wing unmanned aerial vehicle", Fournal of Aircraft, Vol. 52 No. 6.

Qazi, A.I., Ahsan, M., Ashraf, Z. and Ahmad, U. (2017), "Modeling of a UAV longitudinal dynamics through system identification technique", International fournal of Aerospace and Mechanical Engineering, Vol. 11.

Sobolewski, M., Grzesik, N., Koruba, Z. and Nowicki, M. (2016), "Fuzzy logic estimator implemented in observationtracking device control", Aircraft Engineering and Aerospace Technology, Vol. 88 No. 6, pp. 697-706.

Sufendi, Trilaksono, B.R., Nasution, S.H. and Purwanto, E.B. (2013), "Design and implementation of hardware-in-the- loopsimulation for UAV using PID control method", 3rd International Conference on Instrumentation, Communications, Information Technology and Biomedical Engineering (ICICI-BME).

Sushchenko, O.A. and Shyrokyi, O.V. (2015), " $\mathrm{H} 2 / \mathrm{H} \infty$ optimization of system for stabilization and control by line-of-sight orientation of devices operated at UAV", IEEE International Conference Actual Problems of Unmanned Aerial Vehicles Developments (APUAVD), Ukraine, pp. 235-238.

Tecktron Systems (2009), "Shadow 400", available at: www. uvsr.org/docs/AAI_USA_Shadow-400_2009.pdf

Triputra, F.R., Trilaksono, B.R., Sasongko, R.A. and Dahsyat, M. (2012), "Longitudinal dynamic system modeling of a fixed-wing UAV towards autonomous flight control system development - a case study of BPPT Wulung UAV platform", International Conference on System Engineering and Technology (ICSET).

Tristancho, J., Quilez, M. and Pastor, E.E. (2010), “An electromagnetic interference reduction check list for unmanned aircraft system", 48th AIAA Aerospace Sciences Meeting Including the New Horizons Forum and Aerospace Exposition, Orlando, FL.

\section{About the authors}

Eduard Bertran received the engineer and

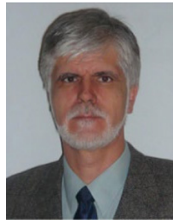
doctor engineer degrees in telecommunication from the Technical University of Catalonia (UPC), Barcelona, Spain, in 1979 and 1985, respectively. In 1987, he joined the department of Signal Theory and Communications (TSC), where he has been the head of studies, and he is currently a full professor. His research interests include control theory, signal processing, radio-communication electronics and avionics. He has served as an associate director for aeronautical studies at the Castelldefels School of Telecommunications and Aerospace Engineering - EETAC. $\mathrm{He}$ has been the coordinator of the doctorate program in aerospace science and technology. He has collaborated in different national and European research projects (including Capanina and HeliNet projects on high altitude platforms) and teams, and he has also collaborated in industrial projects. $\mathrm{He}$ is the author or coauthor of a number of journals and conference papers, as well as books and book chapters. Eduard Bertran is the corresponding author and can be contacted at: eduard.bertran@upc.edu

Paula Tercero Chulián was born in 1996 and received the MS in aerospace engineering from the UPC in 2018. During last years, she has been working in a company devoted to the exploitation of UAVs. She is currently following a master in robotics and automation, and she is interested in UAVs design, control and utilization.

Alex Sànchez-Cerdà was born in Barcelona in

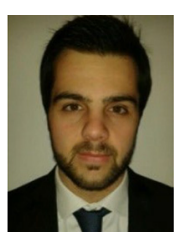
1992. He received the MS degree in aerospace engineering from the UPC in 2014. During his studies, he held a grant from the Government of Catalonia. $\mathrm{He}$ is currently working in a private company as a consultant in software engineering and participating in the Zeus project. His research interests are aerospace, UAVs, control strategies and avionics. 